\title{
Bismuth- and Lithium-Loaded Plastic Scintillators for Gamma and Neutron Detection
}

\author{
Nerine J. Cherepy ${ }^{\mathrm{a},{ }^{*}}$, Robert D. Sanner ${ }^{\mathrm{a}}$, Patrick R. Beck ${ }^{\mathrm{a}}$, Erik L. Swanberg ${ }^{\mathrm{a}}$, \\ Thomas M. Tillotson ${ }^{\mathrm{a}}$, Stephen A. Payne ${ }^{\mathrm{a}}$ and Charles R. Hurlbut ${ }^{\mathrm{b}}$ \\ ${ }^{a}$ Lawrence Livermore National Laboratory, Livermore, $C A$ \\ ${ }^{b}$ Eljen Technology, Sweetwater, TX
}

\begin{abstract}
Transparent plastic scintillators based on polyvinyltoluene (PVT) have been fabricated with high loading of bismuth carboxylates for gamma spectroscopy, and with lithium carboxylates for neutron detection. When activated with a combination of standard fluors, 2,5-diphenyloxazole (PPO) and tetraphenylbutadiene (TPB), gamma light yields with $15 \mathrm{wt} \%$ bismuth tripivalate of $5000 \mathrm{Ph} / \mathrm{MeV}$ are measured. A PVT plastic formulation including $30 \mathrm{wt} \%$ lithium pivalate and $30 \mathrm{wt} \%$ PPO offers both pulse shape discrimination, and a neutron capture peak at $\sim 400 \mathrm{keVee}$. In another configuration, a bismuth-loaded PVT plastic is coated with $\mathrm{ZnS}\left({ }^{6} \mathrm{Li}\right)$ paint, permitting simultaneous gamma and neutron detection via pulse shape discrimination with a figure-of-merit of 3.8, while offering gamma spectroscopy with energy resolution of $\mathrm{R}(662 \mathrm{keV})=15 \%$.
\end{abstract}

Keywords: plastic scintillator, gamma plastic, neutron plastic, neutron capture scintillator, phoswich, pulse-shape discrimination

*Corresponding author.

E-mail address: cherepy1@1lnl.gov

Telephone: 925-424-3492 


\section{Introduction}

Plastic scintillators are widely deployed for ionizing radiation detection, as they can be fabricated in large sizes, for high detection efficiency, however commercial plastics are limited in use for gamma spectroscopy. Detecting and identifying weak radioactive sources within natural background requires large detector volumes in systems offering sufficient gamma ray spectroscopy, as well as the capability to detect neutrons and to distinguish them from gamma rays. At present, the cost of large-size detectors providing accurate radioisotope identification is limited by the cost of the raw materials, growth and fabrication of the detector materials; most typically based on single crystal Thallium-doped Sodium Iodide, NaI(Tl), for gamma spectroscopy, and Helium-3 gas proportional counters for neutron detection. Unfortunately, standard plastic scintillators have low effective $\mathrm{Z}\left(\mathrm{Z}_{\mathrm{eff}} \approx 4.5\right)$, resulting in extremely poor photopeak efficiency (which scales as $Z^{4}$ ), and only a few efforts to use them for gamma ray spectroscopy have been undertaken via energy windowing and reconstruction algorithms that analyze Compton edges, as reported in [1-3]. These approaches require far more events and complex data analysis compared to standard photopeak spectroscopy. Attempts have previously been made to increase $\mathrm{Z}_{\mathrm{eff}}$ by doping with heavy metals to induce a photopeak [1, 4-8]. However, difficulties due to poor solubility of the high-Z component, its quenching of radioluminescence due to spin-orbit coupling, and decomposition of the high- $\mathrm{Z}$ compound into brown-colored products during polymerization make formulating an effective gamma spectroscopy plastic challenging. In our previous work, we described plastics based on polyvinylcarbazole (PVK) loaded with up to $40 \mathrm{wt} \%$ triphenyl bismuth $\left(\mathrm{BiPh}_{3}\right)$, corresponding to $19 \mathrm{wt} \%$ bismuth metal, providing light yields of up to $30,000 \mathrm{Ph} / \mathrm{MeV}$ and with energy resolution $\mathrm{R}(662 \mathrm{keV})<7 \%$ [9, 10]. An alternative class of bismuth compounds with high 
solubility in non-polar organics is the bismuth carboxylates, and the carboxylate compound explored in this paper is bismuth tripivalate (Figure 1).
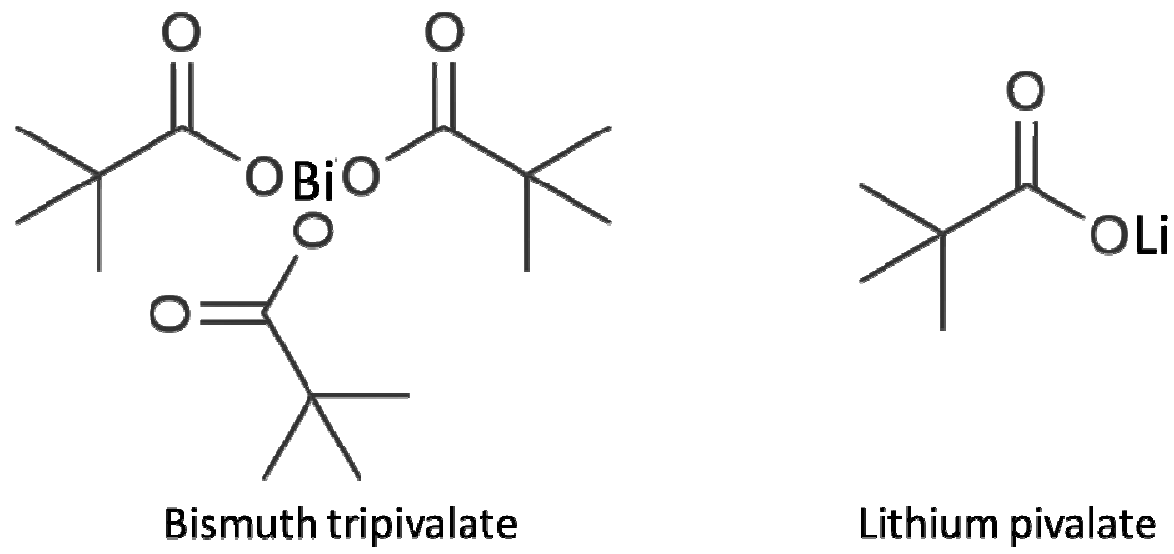

Figure 1. Bismuth and lithium pivalate chemical structures reveal the features that render them highly soluble, capable of high metal loading of plastics, and highly stable in air, as well as in plastic dispersions. First, the t-butyl tail of the ligand is soluble in the non-polar plastic matrix. Second, the carboxylate moiety strongly bonds the metal center, making it resistant to hydrolysis. The overall molecular weight of these complexes is also minimized, compared to other carboxylates, allowing high fractional metals loading in plastics.

Organic scintillators interact with neutrons efficiently via proton recoil (cross section ranges from 4-20 barns in the 0.01-1 MeV range [11]), and organic scintillators with the appropriate formulations (certain liquids, plastics and single crystals) offer pulse shape discrimination (PSD), permitting discrimination between high-energy neutron and gamma interactions $[1,12]$.

Introduction of ${ }^{6} \mathrm{Li}$ into plastic scintillators provides a high cross section, 940 barns, for thermal neutron capture, and a higher Q value compared to other options for thermal neutron capture, such as ${ }^{10} \mathrm{~B}$. Thermal neutron capture on ${ }^{6} \mathrm{Li}$ produces an alpha particle and a triton with total energy, or $\mathrm{Q}$ value, of $4.78 \mathrm{MeV}$, while ${ }^{10} \mathrm{~B}$ produces a ${ }^{7} \mathrm{Li}$ and an alpha with a smaller combined Q value of 2.31 MeV (94\% branching) or $2.79 \mathrm{MeV}(6 \%)$. PSD in Li-loaded scintillators results from the difference in energy deposition rate resulting from high energy electrons (gamma 
absorption), proton recoils (high energy neutron collisions) or the alpha + triton (thermal neutron capture) yielding different amounts of exciton-exciton quenching; this is observable in the difference in the shapes of the scintillation pulses for each type of interaction [13]. Compared to high energy electrons, the shorter range protons, alphas and tritons (in that order) yield a higher concentration of triplet-triplet annihilation leading to Auger upconversion, and thus larger delayed singlet emission components.

Previous studies describing Li-loaded organics have been limited by the lack of solubility of commonly available highly polar Li-containing compounds in the non-polar aromatic matrices needed for efficient scintillation [14-16]. The so-called "triple PSD" phenomenon has been achieved in a variety of B- and Li-loaded scintillators [see, for example, references 16 and 17] where gammas, high-energy neutrons and thermal neutrons can be distinguished from each other. Here, we describe the use of a Li-carboxylate molecule, lithium pivalate (Figure 1), that is soluble in plastic scintillator up to $1.9 \mathrm{wt} \% \mathrm{Li}$, significantly exceeding previously reported Li-loading levels in plastics (e.g. $0.63 \mathrm{wt} \% \mathrm{Li}$ [15]; $0.27 \mathrm{wt} \%$ [16]). We find that Li-pivalate exhibits high solubility, even in a high fluor-loaded plastic scintillator formulation offering PSD, thus providing both thermal and high energy neutron detection and discrimination from gammas.

A variety of inorganic single crystals and glasses based on Li, B or Gd are used for neutron detection. One well-known inorganic scintillator offering excellent discrimination between gammas and thermal neutrons is Cerium-doped Cesium Lithium Yttrium Chloride, or CLYC(Ce) [18], in which neutron capture on ${ }^{6} \mathrm{Li}$ produces a peak at 3.4 MeVee, an energy higher than terrestrial gamma background. CLYC(Ce) also offers gamma spectroscopy with good energy resolution, $\mathrm{R}(662 \mathrm{keV})<6 \%$. The high light yield for the neutron capture event, as well as the good energy resolution are due to the good light yield proportionality of CLYC(Ce). In addition 
to pulse-height discrimination with CLYC(Ce), PSD may be used, as the neutron and gamma events produce distinctive pulse shapes. By comparison, Li-plastics exhibit significant light yield quenching (poorer light yield proportionality) for neutron capture events, greatly reducing the electron-equivalent energy, and resulting in neutron capture peaks in the 400-600 keVee range [this work, 14-16], too low to be used conveniently for pulse-height discrimination alone.

A detector design for "dual" detection of gammas and neutrons can utilize a "phoswich" approach [19], with a gamma-responsive scintillator and a neutron-responsive scintillator that are “sandwiched" (optically coupled) and produce pulses with different decay times, readily distinguished electronically. Phoswich detectors have previously been demonstrated using standard plastic scintillators with an optically-coupled surface coating of the neutron phosphor, $\mathrm{ZnS}\left({ }^{6} \mathrm{Li}\right)[20]$. In this approach, ${ }^{6} \mathrm{Li}$ reacts with thermal neutrons, producing a bright scintillation pulse, from the $4.78 \mathrm{MeV}$ of the neutron capture products of ${ }^{6} \mathrm{Li}$, and the high light yield of $\mathrm{ZnS}(\mathrm{Ag})$, about 76,000 $\mathrm{Ph} / \mathrm{MeV}$; neutron capture in the phosphor thus results in up to 363,000 Photons/event. Herein we describe use of the phoswich configuration to expand the capabilities of the Bi-plastic, so that in addition to providing gamma ray spectroscopy, it efficiently detects and discriminates neutrons, in a single detector. The plastic volume provides a high concentration of protons for neutron thermalization, thereby also permitting efficient detection of high energy neutrons.

\section{Experimental methods}

Chemicals were obtained from Sigma Aldrich and TCI except for the metal pivalates which were prepared from pivalic acid and triphenylbismuth (bismuth tripivalate) or lithium 
hydroxide (lithium pivalate). Plastics were fabricated from vinyl toluene monomers, 2,5diphenyloxazole (PPO, 99\%), and diphenylanthracene (DPA, >98\%) or tetraphenylbutadiene (TPB,$>98 \%$ ) by combining them with bismuth or lithium carboxylates and a free radical initiator, in a nitrogen-purged glovebox. Chemicals were typically purified by recrystallization and column chromatography. Samples were sealed in glass jars and cured at $70-90^{\circ} \mathrm{C}$ in a nitrogen-purged oven. Once solid, the plastics were removed from the jars for experimentation. Radioluminescence was acquired using a ${ }^{90} \mathrm{Sr} /{ }^{90} \mathrm{Y}$ source $(\sim 1 \mathrm{MeV}$ average beta energy), and spectra were collected with a Princeton Instruments/Acton Spec 10 spectrograph coupled to a thermoelectrically cooled CCD camera. Gamma pulse-height spectra were measured with a ${ }^{137} \mathrm{Cs}$ source, while neutron detection properties were studied using a ${ }^{252} \mathrm{Cf}$ source shielded with 2 inches of lead, which reduces the gamma rates to the same order of magnitude as neutrons, and 3 inches of polyethylene, which increases the ratio of thermal to fast neutrons. Scintillators were optically coupled to a Hamamatsu R6231-100 photomultiplier tube, which was connected to an Ortec 113 preamplifier. The signals were shaped with a Tennelec TC 243 spectroscopy amplifier (shaping time typically $1 \mu \mathrm{s}$ ) and then recorded with an Amptek MCA8000-D multichannel analyzer. Effective light yields were determined by reference to a standard plastic from Eljen Technology, EJ-200, and energy resolution, full width at half maximum (FWHM), obtained by non-linear least-squares fitting of peaks in Igor Pro software.

For digital readout, used for PSD measurements, scintillation pulses were collected with a Bridgeport Instruments eMorpho 12 bit waveform digitizing ADC with $80 \mathrm{MHz}$ digitization, 1024 point waveform capture via FPGA that operates on $<0.5 \mathrm{~W}$. Neutron-gamma pulse shape discrimination with a ${ }^{252} \mathrm{Cf}$ source was evaluated using the $\mathrm{Q}_{\text {Tail }} / \mathrm{Q}_{\text {Total }}$ PSD discriminator. The waveforms were numerically integrated over two time intervals: $\Delta \mathrm{t}_{\text {Total }}$ and a subinterval $\Delta \mathrm{t}_{\text {Tail }}$, 
corresponding to the total charge and the delayed component of the signal respectively, where $\Delta \mathrm{t}_{\text {Tail }}$ was optimized to achieve the best discrimination between neutron and gamma events for a given set of pulses. A custom algorithm was used to select the optimal $\mathrm{Q}_{\text {tail }}$ from the fixed integration time of $\mathrm{Q}_{\text {total }}$ of $500 \mathrm{~ns}$, and a pulse shape analysis performed offline to produce PSD plots. Quantitative evaluation of PSD was made using a figure-of-merit $\mathrm{FOM}=\mathrm{S} /\left(\delta_{\mathrm{gamma}}+\delta_{\text {neutron }}\right)$, where $\mathrm{S}$ is the separation between gamma and neutron peaks, and $\delta_{\text {gamma }}$ and $\delta_{\text {neutron }}$ are the FWHM of the corresponding peaks provided by Gaussian fits.

\section{Results and Discussion}

Loading of plastics with metals that enhance their gamma stopping (high Z metals such as Bi) or neutron capture cross sections (such as Li) is complicated by the moderate solubility of commercially available polar metal-containing compounds in the non-polar aromatic matrices needed for efficient scintillation. Metal carboxylates are a diverse class of compounds in which the metal center is bonded to a carboxyl group, the simplest of which is the acetate moiety. A wide variety of carboxylate ligands can be considered, some having long aliphatic chains, such as a caproate group, some with a polymerizable vinyl group, such as a methacrylate; both of these alternatives have recently been suggested by Bertrand and co-workers [10], though plastic scintillator formulations based on these compounds were not found to outperform triphenyl bismuth-loaded plastics. For both bismuth and lithium loading of plastics, we have found excellent air stability and high solubility in aromatic matrices for metal carboxylates based on the pivalate group. The pivalate group offers a short, branched termination of the carboxylate linkage to the metal, in the form of the tertiary butyl (t-butyl) group (see Figure 1). 


\subsection{Bismuth-Loaded Plastics}

In our efforts to obtain high Bi-loading in standard PVT-based scintillator formulations, we have fabricated a range of PVT/Bi-tripivalate plastics and report here on their properties. Figure 2 shows excellent transparency of PVT / Bi-tripivalate plastics, with loading as high as $42 \mathrm{wt} \% \mathrm{Bi}(70 \mathrm{wt} \%$ Bi-tripivalate).

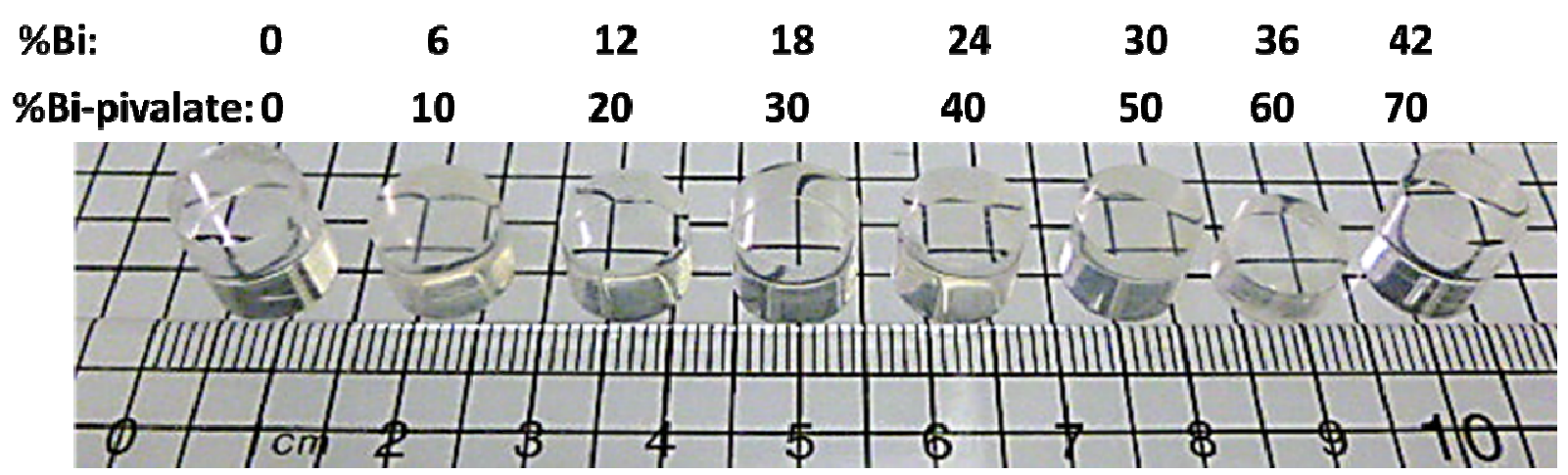

Figure 2. Series of PVT-based samples showing excellent transparency as bismuth tripivalate is added. Loadings of $42 \mathrm{wt} \%$ bismuth metal can be obtained without degrading transparency, due to the high solubility of bismuth tripivalate in the organic matrix.

While Bi-tripivalate offers excellent solubility in PVT, scintillation light yields are quenched as a function of bismuth concentration, as is well-known for high-Z loading of plastic scintillators and generally accepted to be due to spin-orbit coupling reducing the fraction of singlets and increasing the triplet population after the creation of excited states from the initial ionizing radiation [8]. Figure 3A shows the radioluminescence (RL) of PVT-based scintillators activated with typical organic fluors that emit only from the singlet state. PVT with $2 \%$ PPO offers a similar integrated RL to that obtained with PVT with $2 \%$ PPO and $0.1 \%$ DPA, while the DPA emission is better matched to PMT readout and self-absorption by the scintillator is much 
less in the longer wavelength range. In comparison to the RL of the commercial Eljen EJ-200 scintillator, the RL light yield of the LLNL-fabricated plastics is about 50\% lower. Figure 3B shows the RL obtained with a series of PVT scintillators with 2\% PPO and 0.1\% DPA, as a function of added Bi-tripivalate.

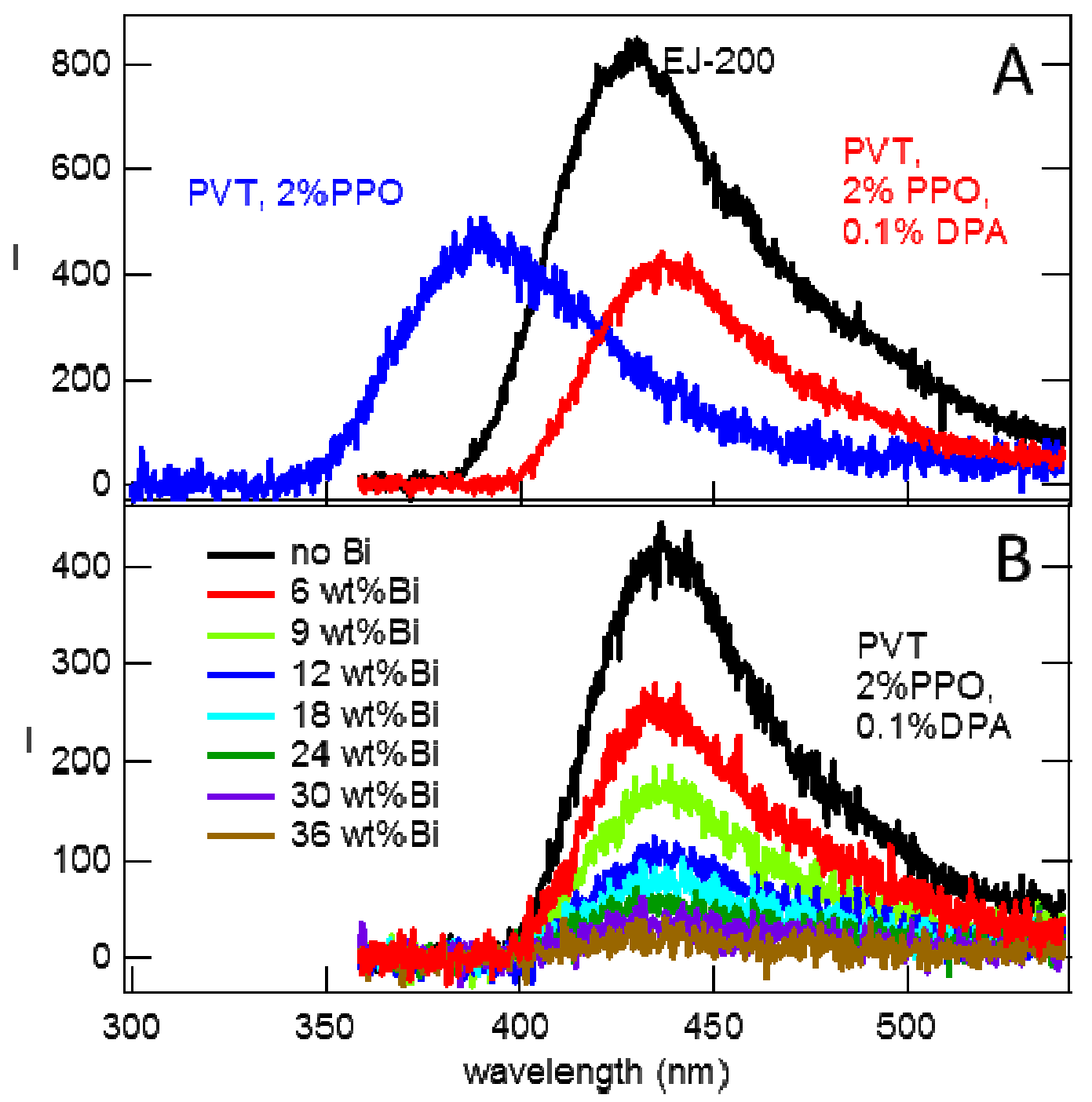

Figure 3. (A) Beta-excited radioluminescence (RL) of LLNL-fabricated plastic scintillators, without any metal loading, compared to commercial Eljen EJ-200 scintillator. (B) Radioluminescence of Bi-tripivalate-loaded scintillators. Light yield decreases as a function of bismuth loading. As in Figure 2, all samples exhibit excellent clarity and homogeneity (not shown). 
In our previous work, polyvinylcarbazole (PVK) was used as the host polymer and triphenyl bismuth $\left(\mathrm{BiPh}_{3}\right)$ as the bismuth constituent. Quenching of RL as a function of bismuth content is observed for both the $\mathrm{PVK} / \mathrm{BiPh}_{3}$ formulations and for the PVT/Bi-tripivalate formulations, though the quenching with Bi-tripivalate is more pronounced at high loadings (Figure 4).

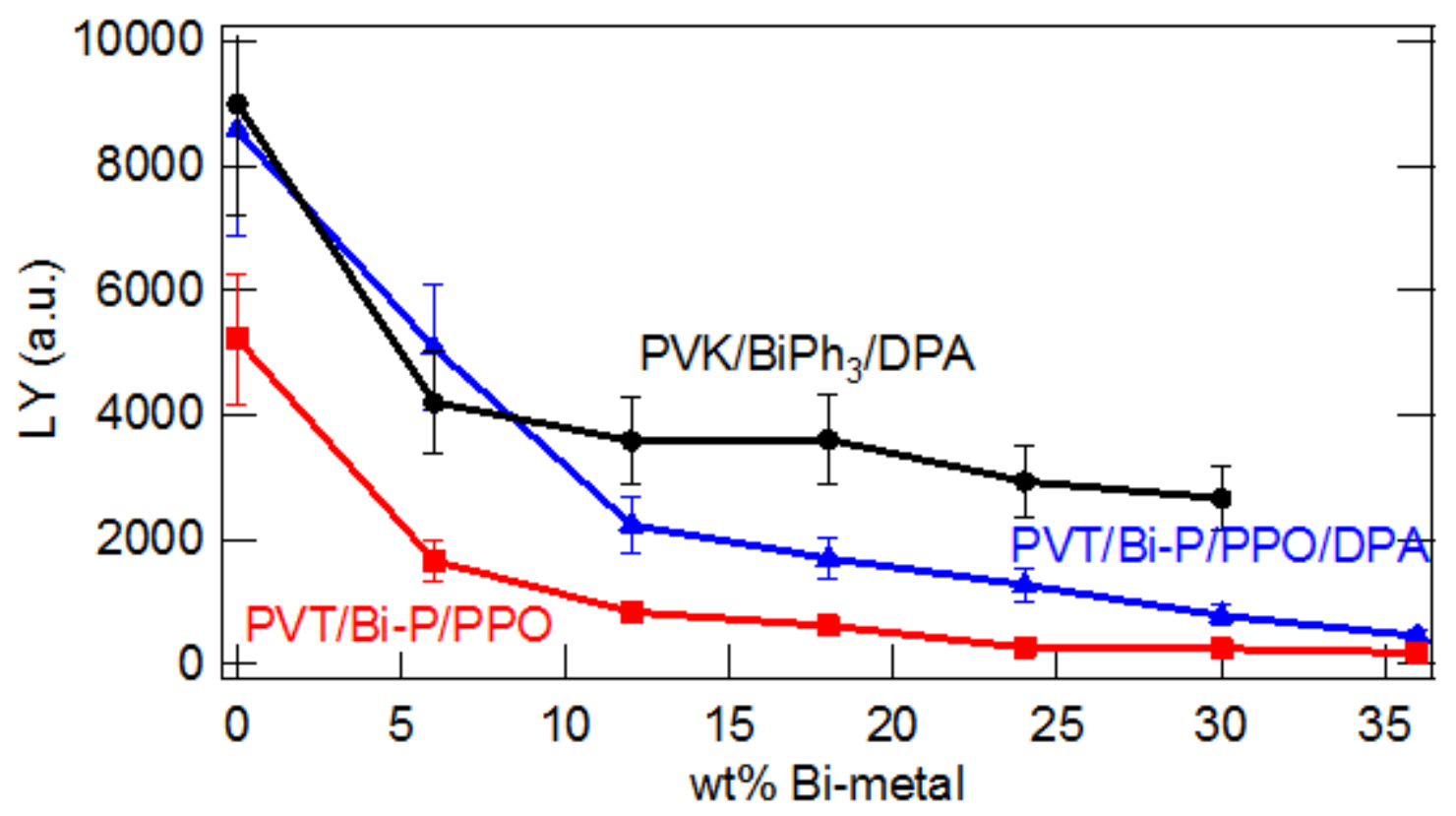

Figure 4. Radioluminescence light yields (integrals of RL spectra) of plastic scintillator compositions based on PVT with Bi-tripivalate (Bi-P) exhibit stronger quenching as a function of bismuth metal concentration than compositions based on polyvinylcarbazole with triphenyl bismuth.

A comparison of bismuth loading levels obtained with several bismuth compounds is shown in Table 1. The maximum loading level is defined by the highest loading achievable while still resulting in an optically transparent scintillator. While scintillators with very high loadings of Bi-tripivalate can be fabricated, the light yield quenching, described in Figure 4, limits their use, 
so far. A $9 \mathrm{wt} \% \mathrm{Bi}$ (15 wt\% Bi-pivalate) formulation was selected for scale up, and is described further, below.

Table 1. Bismuth pivalate is found to have excellent solubility in PVT-based plastic scintillator compositions, allowing for loadings of up to $42 \mathrm{wt} \%$ Bismuth metal.

\begin{tabular}{|c|c|c|c|c|}
\hline $\begin{array}{c}\text { Bismuth } \\
\text { compound }\end{array}$ & $\begin{array}{c}\text { MW } \\
(\mathbf{g} / \mathbf{m o l})\end{array}$ & $\begin{array}{c}\text { Fraction } \\
\text { Bismuth in compound }\end{array}$ & $\begin{array}{c}\text { Maximum loading } \\
\text { by compound }\end{array}$ & $\begin{array}{c}\text { wt\% Bismuth } \\
\text { in scintillator }\end{array}$ \\
\hline Methacrylate $^{\mathrm{a}}$ & 294 & 0.71 & 0.10 & 7 \\
\hline Triphenyl $^{\mathrm{b}}$ & 440 & 0.48 & 0.50 & 24 \\
\hline Pivalate $^{\mathrm{c}}$ & 512 & 0.60 & 0.70 & 42 \\
\hline
\end{tabular}

a. see Ref. 10

b. see Ref. 8

c. this work

An example of how the Bi-tripivalate scintillators can be used for gamma ray spectroscopy is shown in Figure 5, the data acquired with a sample containing $9 \mathrm{wt} \% \mathrm{Bi}$. It has a light yield about one-third that of the commercial plastic scintillator EJ-200, and exhibits a partially resolved photopeak when irradiated by a Cs-137 source. The photopeak includes a strong escape peak from Bi-209 $\mathrm{K} \alpha \mathrm{X}$-rays $(74.8$ and $77.1 \mathrm{keV})$, since the sample is only $2 \mathrm{in}^{3}$ in size. 


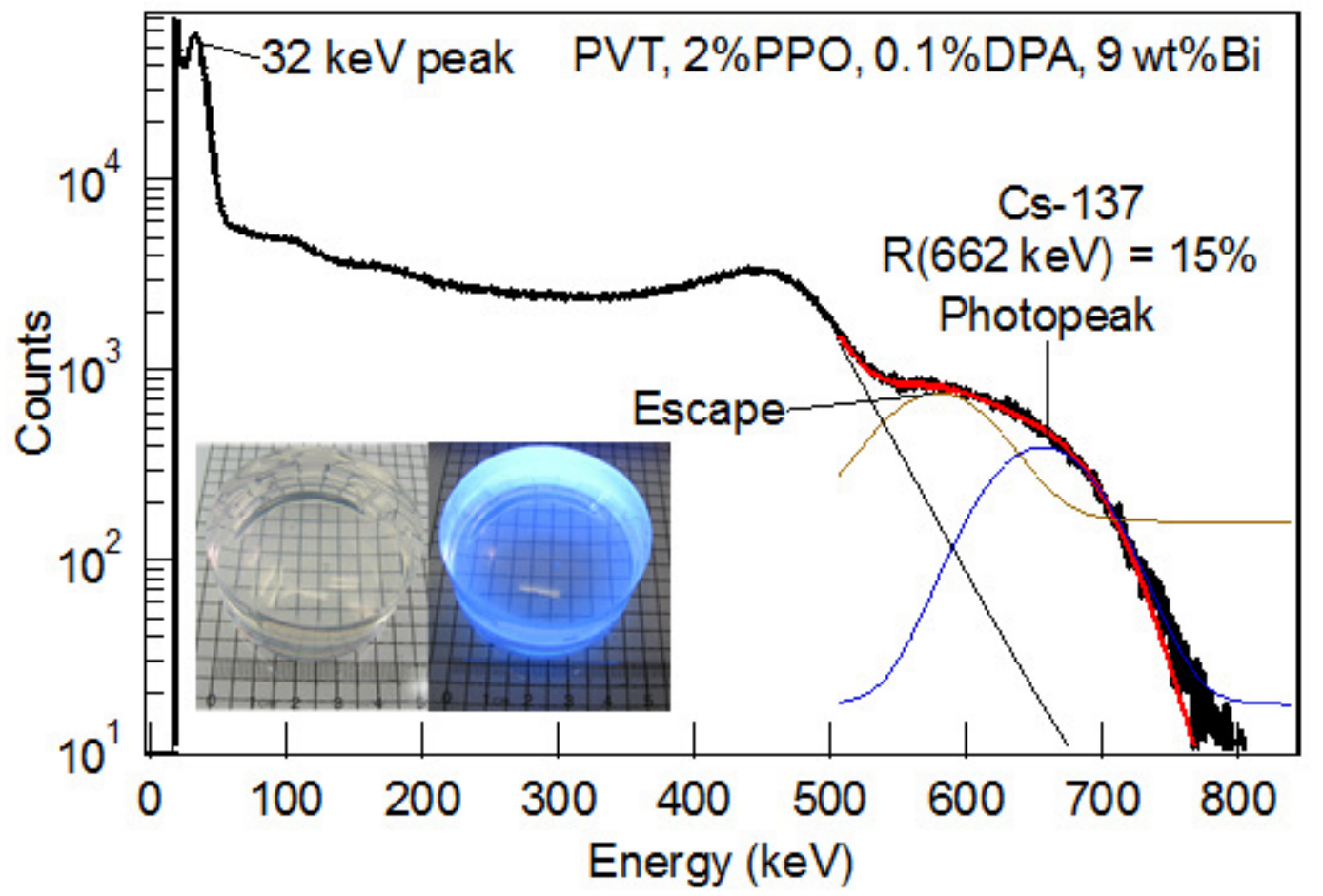

Figure 5. Pulse-height spectrum with Cs-137, acquired with a 2 in $^{3} \mathrm{Bi}-$ loaded plastic, based on PVT matrix, with $15 \%$ Bi-tripivalate, $2 \%$ PPO and $0.2 \%$ TPB. While the photopeak and escape peak overlap, energy resolution of $15 \%$ at $662 \mathrm{keV}$ is obtained, and light yield of $0.35 \mathrm{xEJ} 200$.

\subsection{Lithium-Loaded Plastics}

Similarly to Bi-tripivalate, Li-pivalate is highly soluble in PVT plastic formulations. Figure 6 shows that addition of Li-pivalate does not significantly degrade the light yield of a formulation based on PVT with 2\% PPO. The solubility limit for Li-pivalate in the plastic was found to be $\sim 30 \mathrm{wt} \%$, as depicted in the photo shown in Figure 6, where some Li-pivalate precipitated from the $35 \mathrm{wt} \% \mathrm{Li}$-pivalate sample. 


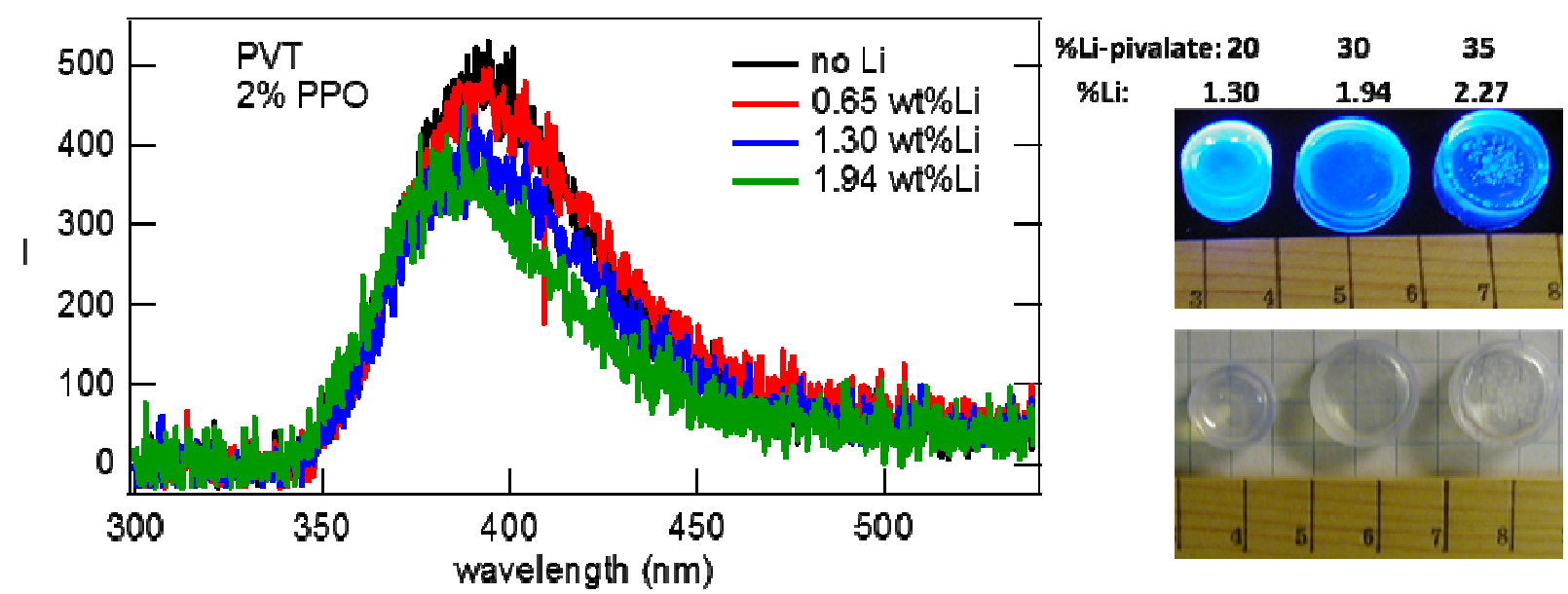

Figure 6. Radioluminescence spectra of PVT / 2\% PPO / Li-pivalate plastics show little effect on light yield with addition of Li-pivalate. The solubility limit of Li-pivalate is around $30 \mathrm{wt} \%$.

Many efforts have been made to load lithium into organics, including liquids, single crystals and plastics. While additional efficiency for neutron capture can be obtained by use of ${ }^{6} \mathrm{Li}$ rather than natural lithium (Nat-Li, contains $7.5 \%{ }^{6} \mathrm{Li}$ ), this adds to the cost of the synthesis, so a pathway to high loading with a Nat-Li, without degrading scintillation properties could ultimately lead to a lower-cost neutron detection material. Alternatively, synthesis and implementation of ${ }^{6} \mathrm{Li}$-pivalate can allow smaller detectors with equivalent neutron capture efficiency to be produced. Table 2 compares the loading achieved with several Li-organics, as well as the inorganic single crystal neutron scintillator, CLYC(Ce). 1.9 wt\% Nat-Li-pivalate can capture $98 \%$ of thermal neutrons with $5 \mathrm{~cm}$ thickness of Nat-Li-pivalate plastic scintillator, while if $1.9 \mathrm{wt} \%{ }^{6} \mathrm{Li}$-pivalate plastic is employed, $98 \%$ of thermal neutrons can be captured with a $0.375 \mathrm{~cm}$ thick plastic. An advantage of a $\sim 5 \mathrm{~cm}$ x $5 \mathrm{~cm}$ Nat-Li-pivalate plastic, such as the one shown in Figure 7, is that the large volume of plastic permits fast neutrons to thermalize and thus be efficiently captured by the Li throughout the volume. In Figure 7, pulse-height spectra 
obtained with a gamma-only source, Cs-137, is compared with that acquired with Cf-252 (shielded with 2" lead), which produces both gammas and neutrons. A pronounced neutron capture peak is observed at $583 \mathrm{keVee}$ for the $30 \mathrm{wt} \%$ Nat-Li-pivalate plastic that does not provide PSD (low-fluor formulation), and at $405 \mathrm{keVee}$ for a high-fluor formulation of PSD plastic with $20 \mathrm{wt} \%$ Nat-Li-pivalate.

Table 2. Lithium pivalate is found to have excellent solubility in PVT-based plastic scintillator compositions, allowing for loadings of up to $1.9 \mathrm{wt} \%$ Lithium metal.

\begin{tabular}{|c|c|c|c|c|}
\hline $\begin{array}{c}\text { Lithium } \\
\text { compound }\end{array}$ & $\begin{array}{c}\text { MW } \\
\text { (g/mol) }\end{array}$ & $\begin{array}{c}\text { Fraction } \\
\text { Lithium in compound }\end{array}$ & $\begin{array}{c}\text { Maximum loading } \\
\text { by compound }\end{array}$ & $\begin{array}{c}\text { wt\% Lithium } \\
\text { in scintillator }\end{array}$ \\
\hline Methacrylate $^{\mathrm{a}}$ & 92 & 0.076 & 0.10 & 0.8 \\
\hline Phenylsalicylate $^{\mathrm{b}}$ & 220 & 0.032 & 0.10 & 0.3 \\
\hline Pivalate $^{\mathrm{c}}$ & 108 & 0.065 & 0.30 & 1.9 \\
\hline CLYC(Ce) $)^{\mathrm{d}}$ & 574 & 0.012 & 1.00 & 1.2 \\
\hline
\end{tabular}

a. See Ref. 15

b. See Ref. 16

c. This work.

d. See Ref. 18 


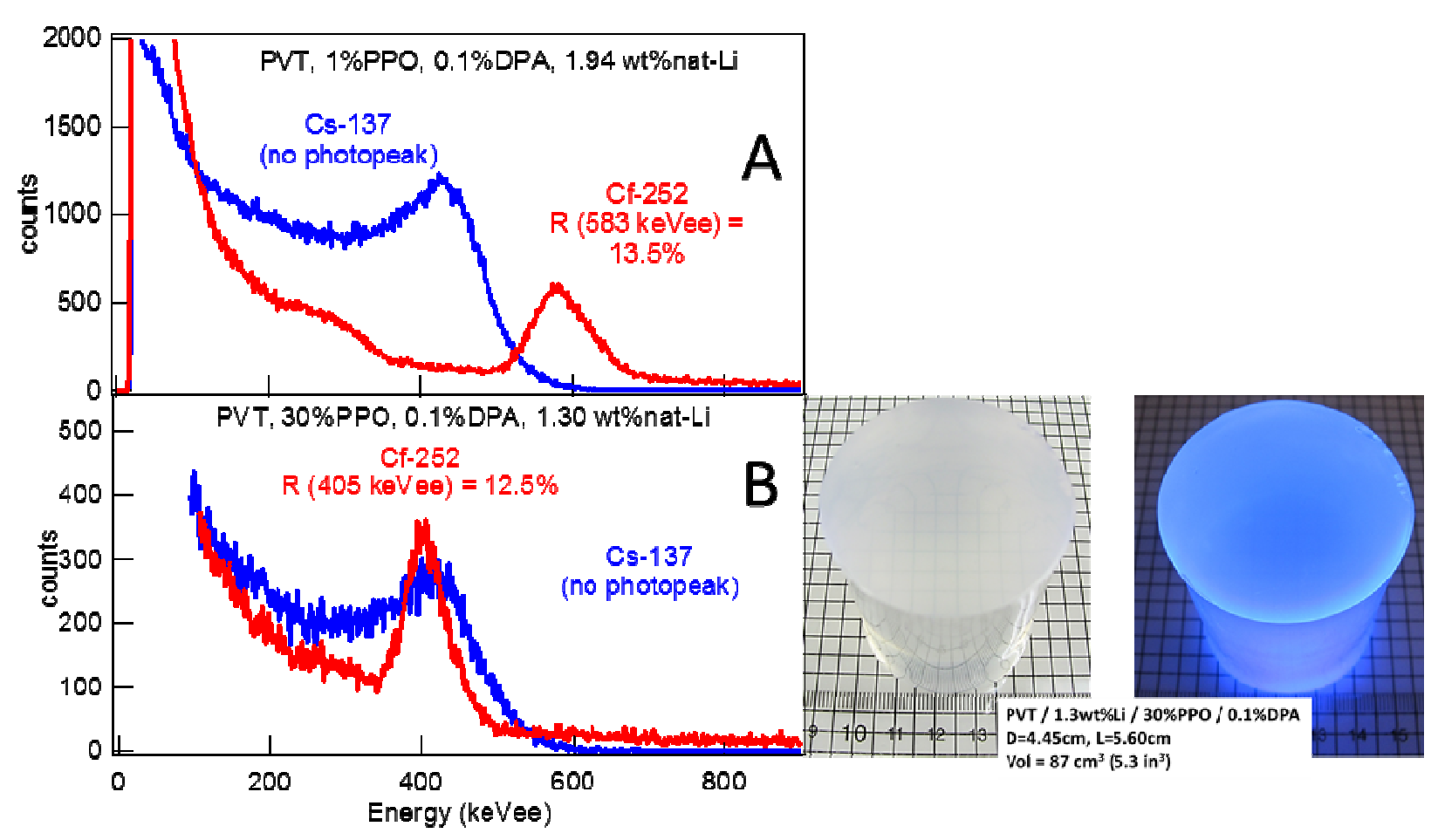

Figure 7. Pulse-height spectra obtained with Cs-137 and Cf-252 sources, with two Nat-Lipivalate-loaded PVT plastics. For the Cf-252 measurements, 2" of intervening lead is used to decrease the gamma event rate. (A) Plastic formulation with typical fluor concentrations used in standard commercial plastic scintillators, $1 \%$ primary fluor (PPO) and $0.1 \%$ secondary fluor (DPA), in which $30 \mathrm{wt} \%$ Li-pivalate can be dissolved (1.94 wt\% Li). (B) A plastic formulation that offers PSD, by employing a high concentration of PPO fluor.(30 wt $\%$ ), along with $0.1 \%$ DPA and $20 \mathrm{wt} \%$ Li-pivalate $(1.3 \mathrm{wt} \% \mathrm{Li})$.

Lithium-loaded plastic scintillator can provide a robust means of detecting neutrons in a high gamma background, if the digitized pulse traces are analyzed, either off-line or on-the-fly, to classify events as gamma, high energy neutron, or thermal neutron based on their pulse shapes. We employed a Bridgeport 12-bit waveform digitizing ADC, and developed a custom algorithm to select the optimal $\mathrm{Q}_{\text {tail }}$ from the fixed integration time of $\mathrm{Q}_{\text {total }}$ of $500 \mathrm{~ns}$. Figure 8 shows that while the thermal neutron events appear strongly clustered around $400 \mathrm{keVee}$, their pulse shape is not distinct enough from the proton recoil events to discriminate thermal neutron events from 
high energy neutron events, though both thermal and high energy neutron events are wellseparated from gamma events above $\sim 300$ keVee.

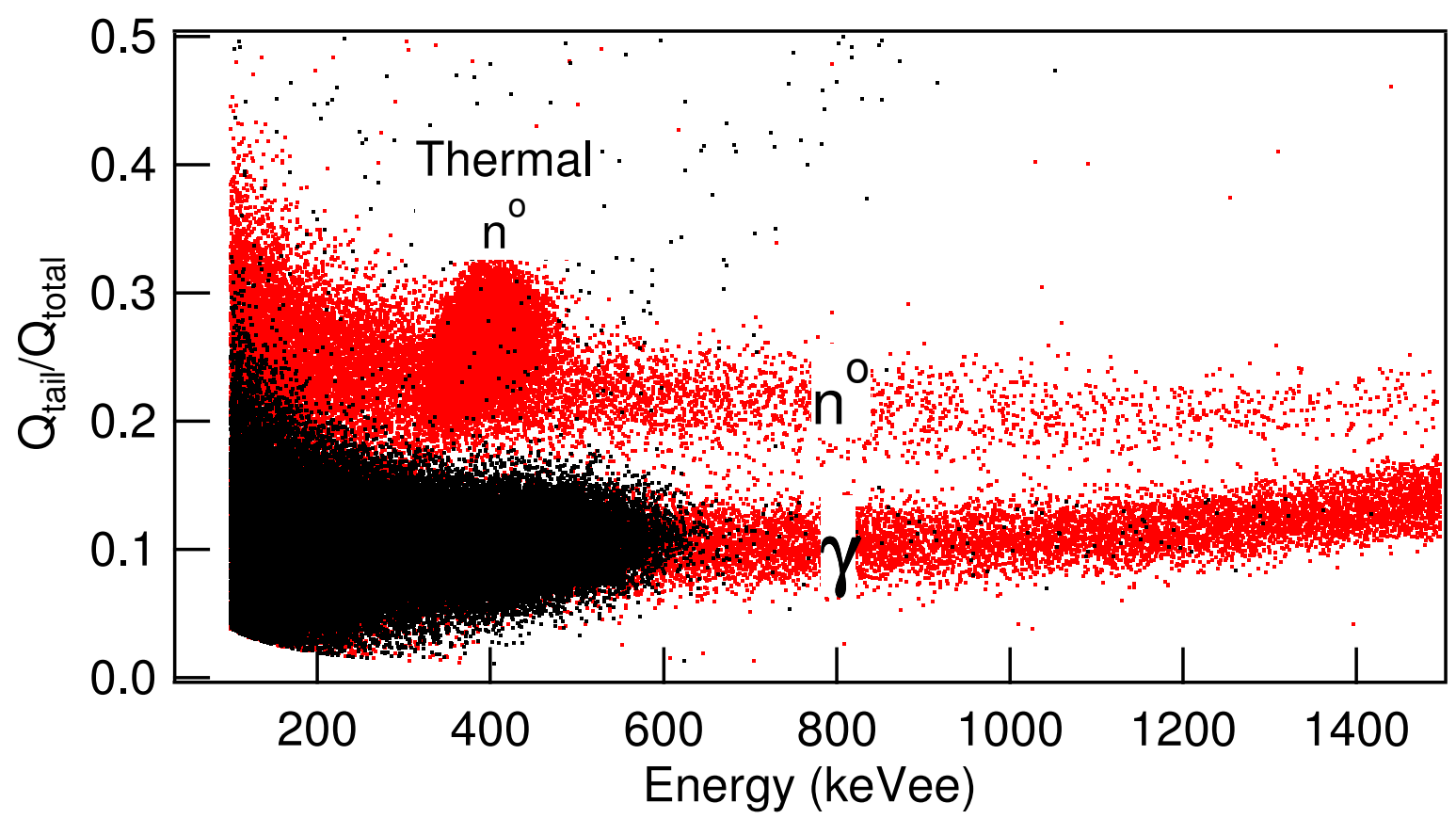

Figure 8. PSD data acquired with the scintillator shown in Figure 7B. Cf-252 (neutron and gamma) events are shown as red dots, while Cs-137 (gamma only) events are shown as black dots. Neutron-gamma discrimination is obtained, and evidence of the neutron capture peak at $\sim 400 \mathrm{keVee}$ and slightly higher $\mathrm{Q}_{\text {tail }} / \mathrm{Q}_{\text {total }}$ is present.

Depending on the energy range selected, the derived FOM for gamma/neutron discrimination varies somewhat. The plots in Figure 9 show that the best FOM, 1.4, is obtained, as expected, over the energy range where the thermal neutron capture peak is present (350-450 keVee), since the thermal neutron capture events are slightly displaced to a higher $\mathrm{Q}_{\text {tail }} / \mathrm{Q}_{\text {total }}$ ratio compared to proton recoils. For the full energy range (all events) the FOM is about 1.3. These values are comparable to those obtained with commercial PSD plastic (without Li), measured to be $\sim 1.5$ in 
reference [21], and represent a first demonstration of Li-based plastic scintillators based on Lipivalate.
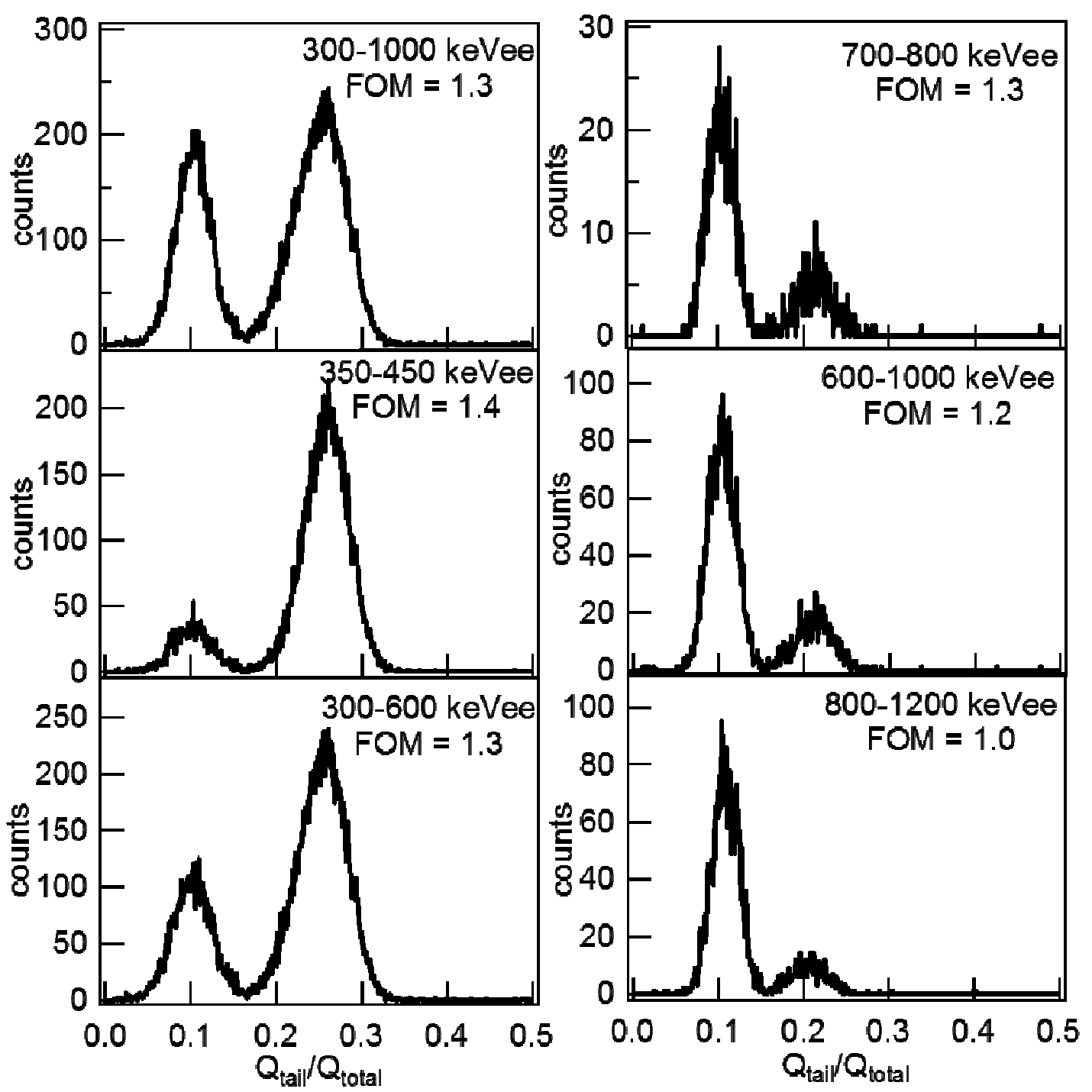

Figure 9. Depending on the energy cut used to analyze the PSD plot shown in Figure 8, histograms covering different energy ranges show variation in the FOM for discrimination between gammas and neutrons. 
A phoswich configuration is an effective approach to large volume scintillation detection of gammas and neutrons in a single detector, based on the use of two optically coupled scintillators: a gamma-responsive scintillator with a particular decay time and a neutron-responsive scintillator with a significantly different decay time. Here, we employed the Bi-plastic scintillator for gamma detection, and a $\mathrm{ZnS}\left({ }^{6} \mathrm{Li}\right)$ coating was used for neutron detection. We painted the Bi-plastic shown in Figure 5 with 200 microns of EJ-424 paint from Eljen Technology, similar to a design described using a standard plastic scintillator by Kiff, et al. [20]. The phoswich detector demonstrated here takes advantage of the fast decay of the Bi-plastic (2 ns decay for gammas) and the slower decay of the $\mathrm{ZnS}\left({ }^{6} \mathrm{Li}\right)$ coating (200 ns decay for neutrons), which are readily distinguished electronically. The Bi-Plastic additionally serves as an effective neutron moderator, thereby eliminating the need for a separate plastic to serve this purpose. This design additionally permits construction of a large volume detector, read out with a single PMT and single electronics channel. It is important to note that the energy axis is calibrated by the light yield of the Bi-loaded scintillator, which is significantly lower than that of the $\mathrm{ZnS}(\mathrm{Ag})$ phosphor light yield, nevertheless, it is useful to see the data as acquired by the system, calibrated to the Bi-plastic. While a $\mathrm{ZnS}\left({ }^{6} \mathrm{Li}\right)$ coating with perfectly uniform distribution of ${ }^{6} \mathrm{LiF}$ and $\mathrm{ZnS}(\mathrm{Ag})$, and with perfect uniformity of optical collection would offer a distinct peak corresponding to neutron captures, this very simple design results in a wide variation of pulse heights associated with neutron captures in the coating, as evidenced by a band of neutron capture events at high $\mathrm{Q}_{\text {tail }} / \mathrm{Q}_{\text {total }}$, rather than a distinct spot with a uniform pulse height. An effective FOM of 3.8 calculated from a histogram of $\mathrm{Q}_{\text {tail }} / \mathrm{Q}_{\text {total }}$ for all events suggests that the chance of misclassifying gamma and neutron events for this type of detector is very small. 

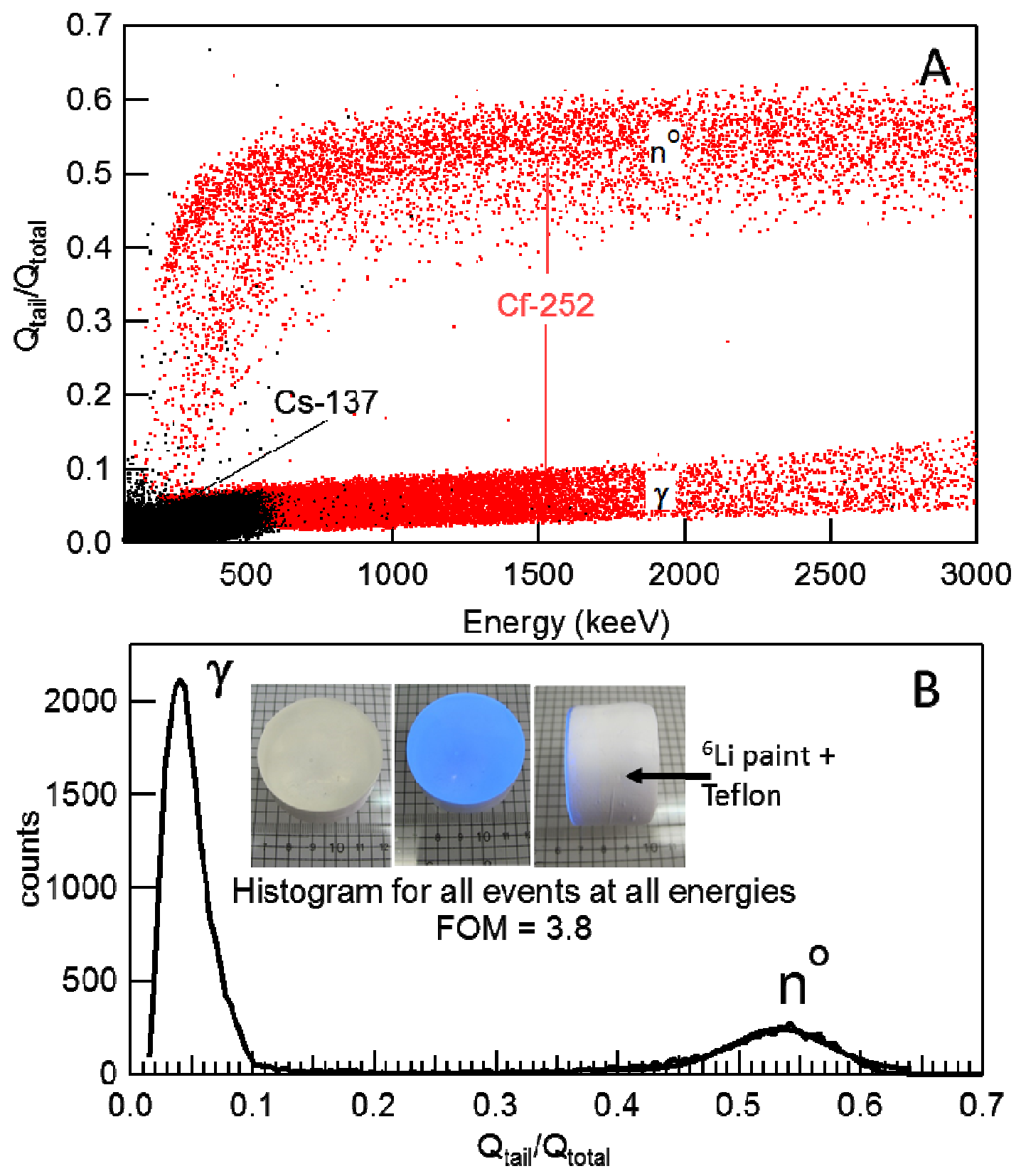

Figure 10. The Bi-plastic sample shown in Figure 5 was painted with 200 microns of $\mathrm{ZnS}\left({ }^{6} \mathrm{Li}\right)$ paint from Eljen Technology, then wrapped in Teflon tape. (A) PSD data reveal excellent discrimination between neutron and gamma events. Cf-252 (neutron and gamma) events are shown as red dots, while Cs-137 (gamma only) events are shown as black dots. The wide range of effective pulse-heights in the neutron band is due to imperfect optics of the neutron-sensitive coating, but the very different decays for neutron and gamma events make discrimination straightforward and with low probability of misclassification. (B) Histogram for all events acquired with the phoswich detector shown in the photo, indicates an excellent FOM for neutron / gamma discrimination. 
While the metal carboxylates, and in particular, the metal pivalates, offer high solubility and stability in plastic scintillators, several challenges to obtaining optimal performance with these materials need to be met. First, the severe quenching of scintillation in the presence of high- $Z$ constituents, such as Bi-tripivalate, via spin-orbit coupling needs to be mitigated, perhaps by use of a fluor capable of emitting triplets, as in our previous report using an Iridium complex [8]. The Li-pivalate-loaded plastics reported here are only the first attempts to formulate a highperformance plastic based on this metal complex, and likely PSD and light yield can be improved by optimizing the concentrations and purity of the constituents. Capture-gated neutron spectroscopy [22], in which one or more proton recoils followed by thermal neutron capture are correlated in order to estimate the fast neutron energy and discriminate against gamma events, is another possible use for a detector using the Li-pivalate plastic.

The phoswich configuration offers a low-cost, robust approach to dual neutron-gamma detection and discrimination, for a wide range of uses. If the Bi-plastic formulation can be produced in large volumes, while also being improved to offer both high $\mathrm{Z}_{\mathrm{eff}}$ and high enough light yield to achieve energy resolution approaching that of Thallium-doped Sodium Iodide, a variety of applications in gamma and neutron detection might be addressed. For example, radiation portal monitors employing a Bi-plastic phoswich detector would provide gamma spectroscopy and neutron detection in a single detector, at a cost similar to the currently employed standard plastic scintillators that only provide gross counting. 


\section{Conclusions}

We have found that Bismuth tripivalate and Lithium pivalate are stable, highly soluble complexes that may be incorporated into PVT plastic formulations up to $42 \mathrm{wt} \%$ Bi or $1.9 \mathrm{wt} \%$ $\mathrm{Li}$, respectively. A Bi-tripivalate plastic with $9 \mathrm{wt} \% \mathrm{Bi}$ was found to provide $\mathrm{R}(662 \mathrm{keV})=$ $15 \%$, in sizes of up to $2 \mathrm{in}^{3}$. A Li-pivalate plastic with $1.3 \mathrm{wt} \% \mathrm{Li}$ and high primary fluor concentration of $30 \%$ PPO offers PSD with a FOM of $\sim 1.3$, and a distinct feature corresponding to neutron capture by ${ }^{6} \mathrm{Li}$ is observed at $405 \mathrm{keVee}$. A Li-pivalate plastic with $1.9 \mathrm{wt} \% \mathrm{Li}$ and standard fluor concentrations (1\% PPO, 0.1\% DPA) exhibits a neutron capture feature at 583 keVee. A prototype phoswich detector based on a Bi-pivalate plastic with a thin coating of $\mathrm{ZnS}\left({ }^{6} \mathrm{Li}\right)$ paint exhibits dual neutron / gamma detection and discrimination with a FOM of 3.8, potentially offering an economical pathway to large volume gamma spectroscopy with neutron detection in a single detector.

\section{Acknowledgements}

This work was supported by the National Nuclear Security Administration, Defense Nuclear Nonproliferation Research and Development Office of the U.S. Department of Energy under Contract DE-AC03-76SF00098, and was performed under the auspices of the U.S. Department of Energy by Lawrence Livermore National Laboratory under Contract DE-AC52-07NA27344. Release number LLNL-JRNL-663264. 


\section{References}

1. F.D. Brooks, "Development of organic scintillators", Nucl. Instr. Methods 162, 477-505 (1979).

2. C. Burt, D. Ramsden, "The Development of Large-area Plastic Gamma-ray Spectrometers," IEEE NSS. Conf. Record, 1186, (2008).

3. J. Ely, R. Kouzes, J. Schweppe, E. Siciliano, D. Strachan and D. Weier, "The use of energy windowing to discriminate SNM from NORM in radiation portal monitors," Nucl. Instrum. Methods A, 560, 373 (2006).

4. S. R. Sandler and K. C. Tsou, "Evaluation of Organometallics in Plastic Scintillators Toward Gamma-Radiation,” Int. J. Appl. Radiat. Isotop. 15, 419 (1964).

5. E.E. Baroni, S.F. Kilin, T.N. Lebsadze, I.M. Rozman, V.M. Shoniya, "Addition of Hetero-Organic Compounds to Polystyrene," Soviet Atomic Energy, 17, 6, 1261-1264 (1964).

6. L.A. Eriksson, C.M. Tsai, Z.H. Cho, C.R. Hurlbut, "Comparative Studies on Plastic Scintillators - Applications to Low Energy High Rate Photon Detection," Nucl. Instrum. Meth., 122, 373-376 (1974).

7. G.I. Britvich, V.G. Vasil'chenko, V.G. Lapshin, A.S. Solov'ev, "New Heavy Plastic Scintillators," Instrum. Exper. Tech., 43, 36-39 (2000).

8. B.L. Rupert, N.J. Cherepy, B.W. Sturm, R.D. Sanner, S.A. Payne, "Bismuth-loaded plastic scintillators for gamma-ray spectroscopy," Europhysics Lett., 97, 22002 (2012).

9. N. J. Cherepy, R.D. Sanner, T.M. Tillotson, S. A. Payne, P.R. Beck, S. Hunter, L. Ahle, P.A. Thelin, "Bismuth-Loaded Plastic Scintillators for Gamma Spectroscopy and Neutron Active Interrogation," IEEE Nuc. Sci. Symp. Conf. Record, N41-3 (2012).

10. G. H. V. Bertrand, F. Sguerra, C. Deh'e-Pittance, F. Carrel, R. Coulon, S. Normand, E. Barat, T. Dautremer, T. Montagu and M. Hamel, "Influence of bismuth loading in polystyrene-based plastic scintillators for low energy gamma spectroscopy," J. Mater. Chem., 2, 7304-7312 (2014).

11. G.F. Knoll, Radiation Detection and Measurement, $4^{\text {th }}$ Ed., 574.

12. N. Zaitseva, B.L. Rupert, I. Pawelczak,A. Glenn, H.P. Martinez, L. Carman, M. Faust, N. Cherepy, S. Payne, "Plastic scintillators with efficient neutron/gamma pulse shape discrimination,” Nucl. Instr. Meth. A, 668, 88-93 (2012).

13. Birks, J.B., The Theory and Practice of Scintillation Counting, Pergamon Press, London (1964).

14. C.D. Bass, E.J. Beise, H. Breuer, C. R. Heimback, T.J. Langford, J.S. Nico, "Characterization of a ${ }^{6} \mathrm{Li}$-loaded liquid organic scintillator for fast neutron spectrometry and thermal neutron detection," Appl. Rad. Isotopes 77, 130-138 (2013).

15. R.D. Bruekers, C.M. Bartle, A. Edgar, "Transparent lithium loaded plastic scintillators for thermal neutron detection," Nucl. Instrum. Meth. A, 701, 58-61, (2013).

16. N. Zaitseva, A. Glenn, H.P. Martinez, L. Carman, I. Pawelczak, M. Faust, S. Payne, "Pulse shape discrimination with lithium-containing organic scintillators," Nucl. Instrum. Meth. A, 729, 747-754 (2013).

17. S. Normand, B. Mouandab, S. Haana, M. Louvel, "Discrimination methods between neutron and gamma rays for boron loaded plastic scintillators," Nucl. Instr. Meth. A 484 342-350 (2002). 
18. J. Glodo, R. Hawrami, K.S. Shah, "Development of $\mathrm{Cs}_{2} \mathrm{LiYCl}_{6}$ scintillator,” J. Cryst. Growth, 379, 73-78 (2103).

19. G.F. Knoll, Radiation Detection and Measurement, $4^{\text {th }}$ Ed., 357-358.

20. S. D. Kiff, N. Bowden, J. Lund, D. Reyna, "Neutron detection and identification using $\mathrm{ZnS}: \mathrm{Ag} /{ }^{6} \mathrm{LiF}$ in segmented antineutrino detectors," Nucl. Instrum. Methods A, 652, 412 (2011).

21. S.A. Pozzi, M.M. Bourne, S.D. Clarke, "Pulse shape discrimination in the plastic scintillator EJ-299-33," Nucl. Instr. Meth. A, 723 19-23 (2013).

22. S.D. Jastaniah, P.J. Sellin, "Digital techniques for $\mathrm{n} / \gamma$ pulse shape discrimination and capture-gated neutron spectroscopy using liquid scintillators," Nucl. Instr. Meth. A, 517, 202-210 (2004). 